\title{
Surface Tension Powered Self-Assembly of 3-D Micro-Optomechanical Structures
}

\author{
Richard R. A. Syms, Member, IEEE
}

\begin{abstract}
A new surface micromachining process for surface tension powered self-assembly of silicon-based microstructures is described. Mechanical parts are formed from bonded silicon-oninsulator material and rotated out-of-plane by melting photoresist pads at low temperature. Simple mechanisms that allow accurate control of the final angle are introduced and used to construct fixed $45^{\circ}$ mirrors and scanning mirror assemblies. [435]
\end{abstract}

Index Terms-MEMS, microactuator, micromirror, MOEMS, scanner, self-assembly, 3-D microstructure.

\section{INTRODUCTION}

$\mathbf{M}$ ICROSTRUCTURE self-assembly is becoming known as a process in which fully three-dimensional microelectromechanical systems (MEMS) are created by repositioning and fixing a set of flat parts created by surface processes such as deposition and etching. So far, the parts have mainly been hinged from the substrate and rotated into position. The hinges have been either flexible strips [1]-[4] or polysilicon hinges [5], [6], and assembly has been manual or by in-built actuation.

Powered assembly offers reductions in assembly time and cost, and improvements in yield. In general, such methods may be either serial or parallel, and either one-time or adjustable. Depending on the application, any of these attributes may be advantageous or disadvantageous. For example, for mass assembly of three-dimensional (3-D) structures, a parallel, onetime method is best; for operation of tracking and scanning devices, individual adjustability may be required. Methods used to power rotation to date include electromagnetic force [7], the shrinkage of a polymer in curing [8], electrochemically induced swelling of polymers [9], [10], surface micromachined vibromotors [11], [12], and microengines [13], [14]. Polymer shrinkage (for example) is inherently parallel and one-time, while motor-driven assembly is serial and adjustable.

In the most sophisticated demonstrations to date, the parts have carried optical components arranged as miniature freespace optical systems on a chip, including laser collimators [15]-[19], tunable filters [20], [21], and beam scanners activated by electrostatic actuators [22], [23], thermal bimorphs [24], [25], and thermally rotated polymer-filled joints [26]. Much of this work is described in a recent review of microoptomechanical systems [27].

Manuscript received March 26, 1999; revised August 24, 1999. Subject Editor, S. D. Senturia.

The author is with the Optical and Semiconductor Devices Section, Department of Electrical and Electronic Engineering, Imperial College of Science, Technology, and Medicine, London SW7 2BT, U.K.

Publisher Item Identifier S 1057-7157(99)09604-3.
An alternative process developed by us uses the surface tension of a pad of meltable material to power the rotation. The available torque was first investigated theoretically using simple geometrical analysis [28], and it was shown that surface tension can also hold the parts together during rotation, without the need for a hinge to connect them [29]. Much of the original analysis was recently verified by others using finite element methods [30], [31].

Surface tension self-assembly was first demonstrated using metal and silicon parts, powered by a semicylindrical fillet of molten solder [32]. A similar process using solder balls has also been demonstrated [30], [31]. A more reliable process based on Si parts rotated by borophospho-silicate glass with controllable viscosity was then developed, which could perform multiple axis assembly operations [33]. However, this involved nonstandard, high-temperature processes, the precision of the assembly was poor, and the structures demonstrated had no real function.

Surface tension self-assembly is potentially an extremely attractive method of forming 3-D microstructures. It is similar to the polymer shrinkage process of Ebefors [8] in being a parallel method. However, key advantages are that the rotation mechanism is inherently simpler, smaller, and more easily combined with other processing.

In the polymer shrinkage process, the flexible joint consists of a series of V-grooves that are first etched through a silicon membrane to a stop, and then filled with polyimide. The result is a kind of bimorph. Rotation involves flexing of the structure into the arc of a circle, rather than pivoting one part with respect to another. The need for an etch stop, and the formation of grooves by anisotropic wet chemical etching, mean that the overall process is complex and material-specific. Without a mechanical limiter, the accuracy of one-time assemblies is relatively low and subject to creep. Although limiters have been incorporated, these are relatively crude. Furthermore, the requirement for a number of grooves to obtain sufficient deflection implies that the hinge mechanism is large $(\approx 100-\mu \mathrm{m}$ radius of curvature [8]). It is therefore unsuitable for precision assembly of structures in this size range.

Important goals in the development of surface tension self-assembly are to eliminate nonstandard processing steps to lower the process temperature, to increase the precision and reliability of the rotation, and to apply it to the fabrication of realistic MEMS components. In this paper, we address all of these points. We first present a modified process based on mechanical parts formed by surface micromachining of industry-standard bonded silicon-on-insulator (BSOI) 
TABLE I

Material Combinations of Previous Surface Tension Powered Self-Assembly Processes

\begin{tabular}{l|c|c|c|c}
\hline Material & Process \#1 [32] & Process \#2 [32] & Process \#3 [33] & This work \\
\hline & & & & \\
\hline Mechanical & Ni metal & Boron-doped Si & Boron-doped Si & Bonded Si \\
\hline Sacrificial & Si substrate & Si substrate & Si substrate & Thermal oxide \\
\hline Meltable & Pb-Sn solder & Pb-Sn solder & BPSG & Photoresist \\
\hline
\end{tabular}

material, which are rotated into position by melting a thick photoresist at low temperature. As with our previous processes, just two lithography steps are required. We then introduce new mechanical rotation limiters capable of inherently high precision. Last, we describe the construction of 3-D microoptomechanical structures such as fixed $45^{\circ}$ mirrors and a resonant electrostatic torsional mirror scanner.

The main application of the process described here is in the accurate, mass assembly of fixed 3-D microstructures. Its main advantages are 1) extreme simplicity, 2) small size, and 3) flexibility. The overall process (two masks; one sacrificial and one mechanical layer) is trivial compared with (say) MUMPS. In contrast to motor-driven assembly, vanishingly small chip area is required for the mechanism. However, it relies on no specific material properties such as crystallinity or an etch stop, and therefore could easily be integrated into a polysilicon process.

\section{BSOI SELF-ASSEMBLy PROCESS}

Fig. 1 shows the general geometry of surface tension powered microstructure self-assembly [28]. Fabrication involves the construction of fixed and movable mechanical parts, linked only by pads of meltable material. The movable parts are perforated, and freed by removal of sacrificial material. Three different materials (mechanical, meltable, and sacrificial, respectively) and two patterning steps (for the mechanical parts and hinge driver pads, respectively) are required. Assembly is carried out by melting the pads and rotating movable parts out of plane.

In previous papers [32], [33], different material combinations have been investigated in an attempt to develop a reliable fabrication sequence, as shown in Table I. For example, mechanical parts have been formed both from nickel (electroplated inside a photoresist mold) and boron-doped silicon (fabricated by implantation, drive-in diffusion, and reactive ion etching). Hinge drivers have been formed from lead-tin solder (again, electroplated in a mold) and lowmelting-point sol-gel borophosphosilicate glass [deposited by spin-coating and rapid thermal annealing, or SC-RTA, and patterned by reactive ion etching (RIE)]. In each case, the sacrificial material was the Si substrate itself, removed by bulk micromachining of (100) wafers.

In each case, difficulties were found. The formation of mechanical parts by electroplating required additional metal interlayers, and the use of the aggressive etch ethylene diamine pyrocatechol (EDP) for undercut caused difficulties with adhesion. However, fabrication of parts by boron doping required a very high drive-in temperature $\left(1250{ }^{\circ} \mathrm{C}\right)$. Using either process, a skewed layout, together with a very deep undercut, was needed to detach the parts.
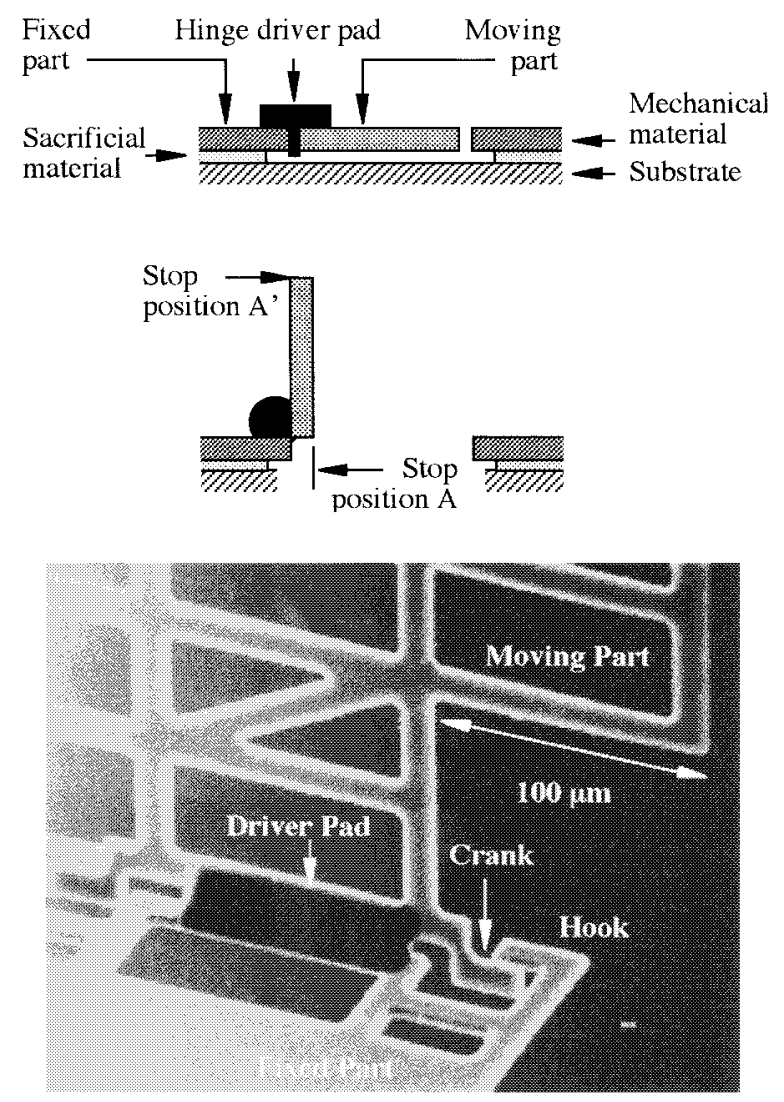

Fig. 1. Top: Schematic of surface tension powered self-assembly [28]. Bottom: Self-assembled structure with substrate-level mechanical limiter, based on bulk micromachined Si parts and borophosphosilicate glass hinge drivers [33].

Similarly, the electroplating of small pads of lead-tin solder with a well-controlled eutectic composition was found to be difficult, and generally resulted in uncontrolled rotation due to the abrupt and poorly defined solid-liquid phase transition. Although extremely controllable composition and viscosity, excellent adhesion, and reliable rotation were obtained using glass driver pads deposited by SC-RTA, a very high melt temperature $\left(1050{ }^{\circ} \mathrm{C}\right)$ was required.

We have now developed a considerably improved surface micromachining process based on BSOI, a material that has recently shown considerable potential for MEMS when combined with deep dry etching [34], [35]. The bonded silicon provides the mechanical parts, while the buried oxide is the sacrificial material, so complicated multilayer processing is entirely avoided. However, the fabrication scheme described here could be adapted to MEMS processes based on polysilicon and silica without difficulty. Since the sacrificial material is now glass, an alternative is required for the hinge drivers. To 


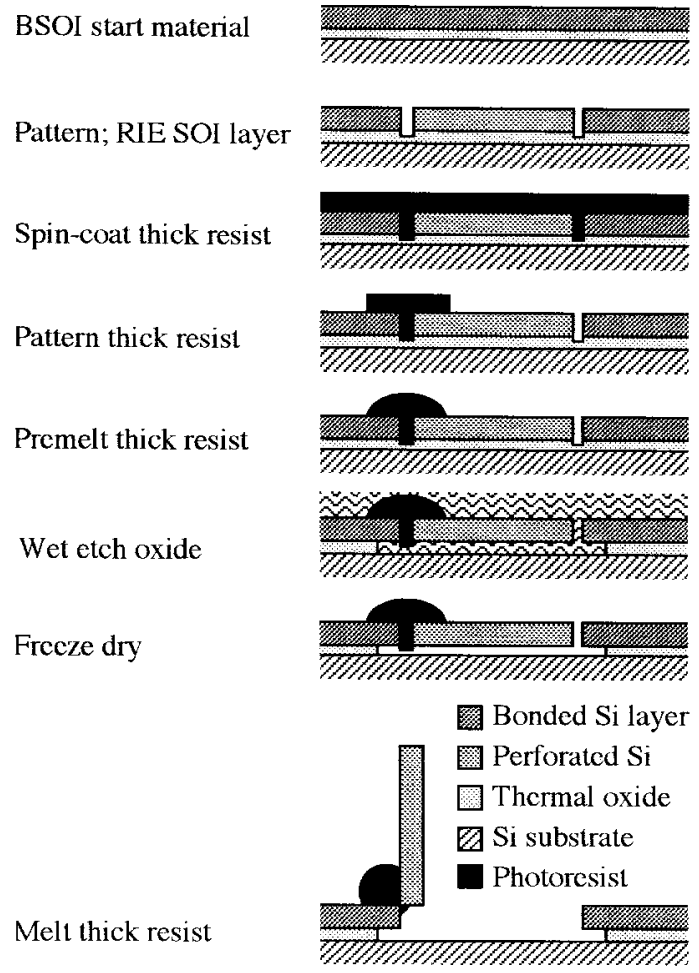

Fig. 2. Process sequence for photoresist-powered self-assembly of BSOI microstructures.

reduce the maximum process temperature, a meltable polymer is an obvious choice. Here, we have used a thick photoresist (Hoechst AZ4562), but photoimagable polyimide is a further possibility.

Fig. 2 shows the new process. Bonded silicon-on-insulator material consisting of 4-in (100) $\mathrm{Si}$ substrates carrying $6-\mu$ m-thick bonded $\mathrm{Si}$ layers on $2-\mu$ m-thick thermal oxide was obtained commercially from BCO Technology (N.I.) Ltd. To define the mechanical parts, a hard mask was first formed from $7000 \AA$ of sputter-deposited $\mathrm{Cr}$ metal, patterned by conventional photolithography and wet chemical etching. This pattern was transferred to the bonded layer by reactive ion etching to a depth of $7 \mu \mathrm{m}$ with a standard parallel plate etcher (Plasma Technology RIE80), using a mixture of $\mathrm{CHF}_{3}$, $\mathrm{O}_{2}$, and $\mathrm{Ar}$ gas. The $\mathrm{Cr}$ mask was then removed by we etching.

The etched wafer was annealed at $900{ }^{\circ} \mathrm{C}$ in $\mathrm{O}_{2}$ for $10 \mathrm{~s}$ to promote adhesion, spin-coated with resist, prebaked at $90{ }^{\circ} \mathrm{C}$, exposed using a Quintel 2001 mask aligner, and developed in Hoechst AZ400K developer (1:4 in DI water) for 6 min. Care was required to determine a suitable resist thickness. Fig. 3 shows the spin-coating and exposure characteristics of AZ4562. The maximum thickness that could be developed out was found to be $h^{\prime} \approx 18.5 \mu \mathrm{m}$, requiring an exposure of $\approx 140 \mathrm{~s}$. To ensure removal of all unwanted resist, the maximum thickness over the surface of the bonded layer was therefore restricted to a value $7 \mu \mathrm{m}$ lower, i.e., $h \approx 11.5 \mu \mathrm{m}$. A spin speed of $1400 \mathrm{rpm}$ was found to be suitable for depositing this thickness. However, since the exposure needed to remove it was only $\approx 50 \mathrm{~s}$, the use of a far longer clearance exposure did lead to some feature degradation.

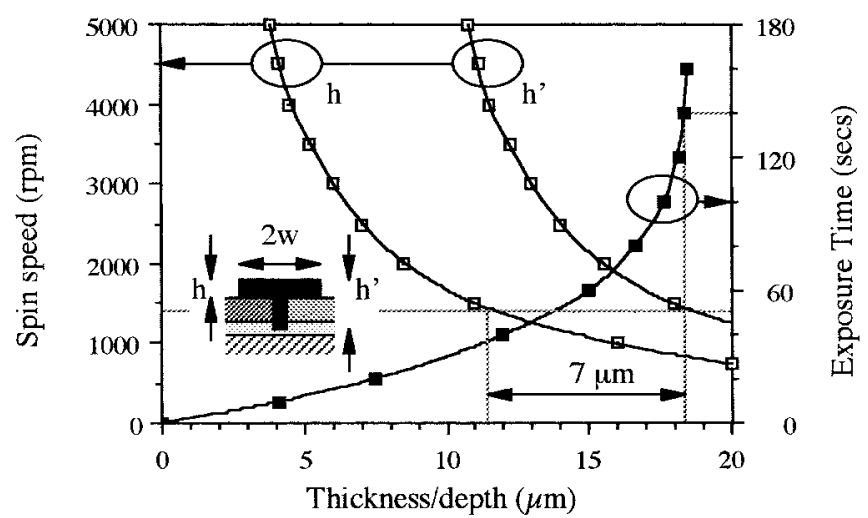

Fig. 3. Spin-coating and exposure characteristic of Hoechst AZ4562 photoresist.

Previous work [28] has shown that the optimum ratio beween the height $h$ of the hinge driver pads and their width $2 w$ for $90^{\circ}$ rotation is $\eta=h / w=(\pi+2) / 8=0.6427$ and that thicker pads yield smaller final rotation angles. To ensure at least $90^{\circ}$ rotation, we therefore used a width of $2 w=36 \mu \mathrm{m}$, corresponding to $\eta=0.639$ for $11.5-\mu \mathrm{m}$ resist thickness. The initial hinge gap was fixed at $2.5 \mu \mathrm{m}$, and the hinge drivers were segmented into $250-\mu \mathrm{m}$ sections.

The dimensions of the movable parts were in the range $250-1000 \mu \mathrm{m}$ measured parallel to the axis of rotation and up to $720 \mu \mathrm{m}$ orthogonal to it. The clearance between all moving parts was fixed at $4 \mu \mathrm{m}$. To free the movable parts, the oxide was removed by etching in $7: 1$ buffered HF, which penetrated through $4-\mu \mathrm{m}$ square holes on a $20-\mu \mathrm{m}$ center-tocenter separation. The duration of this step was determined mainly by the half-width of the hinge driver pads, and for the dimension above was around 7.5 h. To ensure adhesion of the resist pads during such a long etch, they were premelted at $110{ }^{\circ} \mathrm{C}$ for $30 \mathrm{~min}$. After washing, samples were dried in an Edwards Modulyo freeze-drier using a mixture of Aristar methanol and distilled water. Assembly was carried out by melting in an oven at temperatures in the range $130-150^{\circ} \mathrm{C}$, as described later. To improve reflectivity and provide electrical connection across the insulating resist pads, samples were metallized by sputter coating with $500 \AA$ of $\mathrm{Al}$.

\section{MECHANICAL LiMiter FOR ACCURATE $45^{\circ}$ Rotation}

The surface tension obtained when the hinge driver is melted tends to rotate the movable parts out of plane. Originally, it was suggested that the final angle would be determined from a consideration of minimum surface energy, defined by the pad volume [28]. However, two practical considerations-lack of accuracy in defining the volume and lack of a physical link between the fixed and moving parts-make this method unreliable [32]. Instead, a mechanical limiter was adopted in the most recent demonstrations. The limiter was formed together with the fixed and movable parts, so that the overall process complexity was not increased.

In mechanically limited rotation, the movable part is driven against a physical stop by a finite torque. Fig. 1 shows the mechanism used in the first demonstration of this technique [33], which effectively places a stop in the position marked 


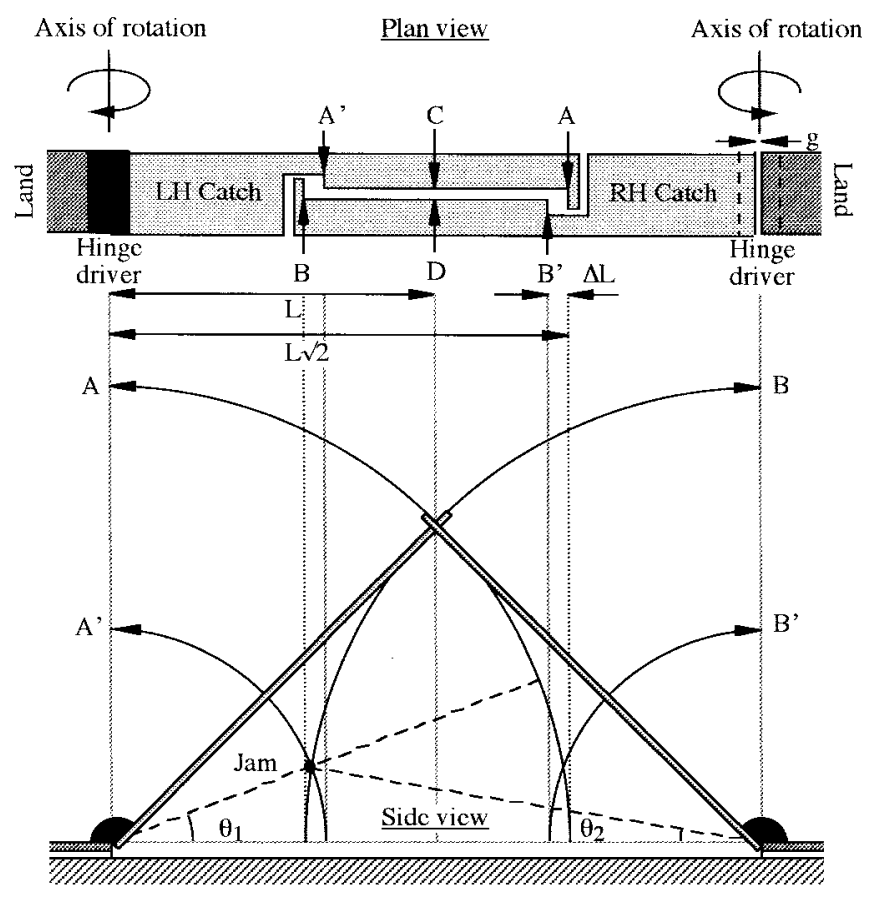

Fig. 4. Layout of above-substrate limiter and geometry of latch and jam conditions.

$A$. The mechanism consists of a crank attached to the movable part, which rotates below the substrate surface to engage with a hook attached to the fixed part. Rotation of $90^{\circ}$ may be achieved for a suitable limiter geometry.

The mechanism is very compact, allowing multiple-axis rotations to be performed easily. However, the sensitivity of the final angle to dimensional errors is high, due to the small lever arm, and the precision achieved was only $\pm 3.75^{\circ}$. To increase accuracy, a longer lever is needed. The minimal clearance obtained by oxide removal suggests that the stop should ideally be above the substrate, where the lever can be maximized, i.e., at $A^{\prime}$ in Fig. 1. An above-substrate spring lock has been developed for the surface micromachined devices described earlier [6], [15], [16]. Improved hinge structures, the most recent of which are self-locking, have also been described [36], [37]. Here, we describe an above-substrate stop for accurate $45^{\circ}$ rotation that may easily be integrated with a surface tension self-assembly process.

The layout of the mechanism is shown in Fig. 4. It consists of two self-assembling flaps, which rotate simultaneously in opposite directions until their extremities catch. The hinges are $2 L$ apart, and the catching surfaces (points $A$ and $B$ ) are $L \sqrt{2}$ from their respective hinges. Assuming symmetry, $A$ and $B$ follow circular trajectories and engage each other when the flaps have rotated through the same angle, which will be $45^{\circ}$ for the dimensions shown. The construction is clearly similar to the classical geometer's method of drawing an isosceles triangle with a pair of compasses.

Two sliding surfaces $C$ and $D$ are provided to guide the catches together when the rotation rates of the flaps are not identical. With this modification, the mechanism will jam through contact between points $A^{\prime}$ and $B$ at the intersection of their trajectories, as shown by the dashed lines in Fig. 4 .
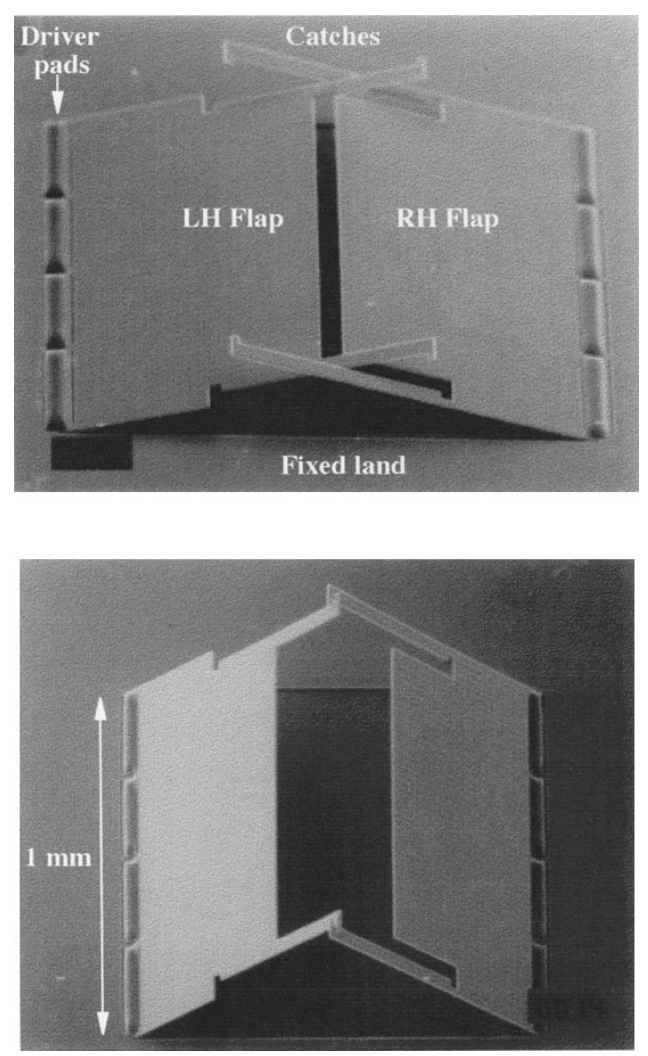

Fig. 5. Self-assembled structures based on surface micromachined BSOI parts and photoresist hinge drivers. Top: Symmetric mirror assembly, partly rotated. Bottom: Symmetric mirror assembly, fully rotated.

There must be clearances $\Delta L$ between $A^{\prime}$ and $B$ so that the mechanism can rotate. However, in the limit of $\Delta L \rightarrow 0$, it is simple to show that the angles of rotation of the flaps are related by $\theta_{1}=\theta_{2} /\{\sqrt{2}-1\}$ at the jam condition, so that their angular velocities must be related by $\omega_{1}=2.414 \omega_{2}$. A similar condition of $\omega_{1}=0.414 \omega_{2}$ exists for $A$ and $B^{\prime}$ to jam. Between these values, $A$ and $B$ are guided together by the surfaces $C$ and $D$. Thus, we might define a "capture range" of $0.414<\omega_{1} / \omega_{2}<2.414$, which suggests a useful tolerance. Outside this range, the guiding mechanism does not engage at all, and the flaps simply miss each other.

Experiments with a macroscopic model broadly validated the argument above. The model was constructed from light metal flaps mounted on freely rotating hinges, and rotation was powered by gravitational force, obtained by releasing the flaps to rotate downwards from a horizontal position. The catches were indeed guided toward each other by the sliding surfaces, so that correct assembly was obtained despite (for example) a delay in releasing one flap, up to a certain limit. However, this implies that the rotation torque was sufficient to overcome sliding friction, and the latter may be relatively more significant in the microstructure size domain.

\section{SELF-ASSEMBLY OF Micro-OPTOMECHANICAL STRUCTURES}

The mechanical limiter of Fig. 4 may be used to construct 3 -D micro-optomechanical components such as $45^{\circ}$ mirrors. Fig. 5 shows scanning electron microscope (SEM) views of 

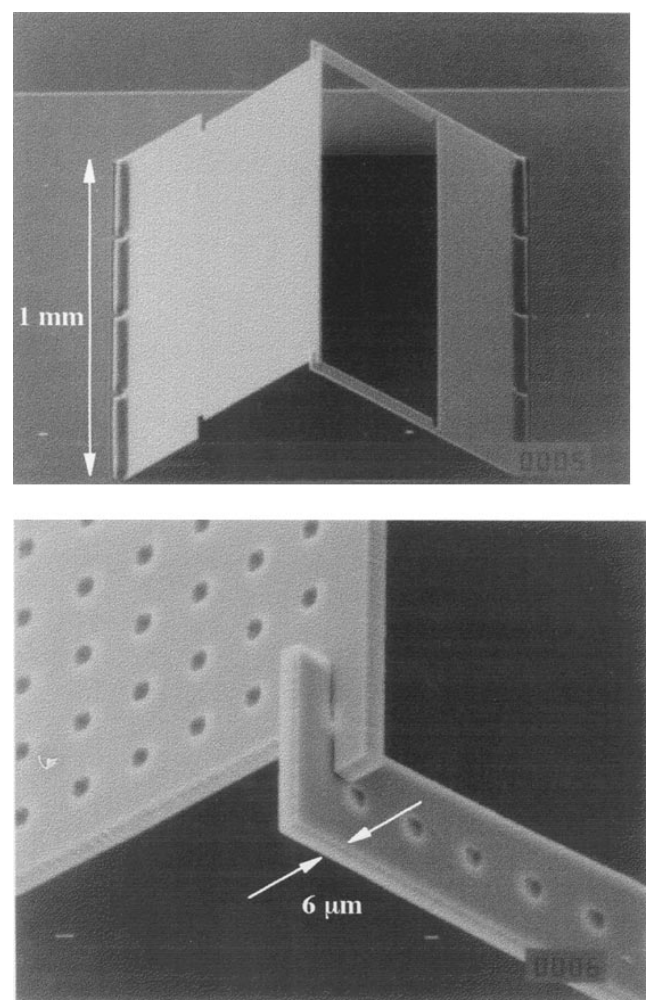

Fig. 6. Self-assembled structures based on BSOI parts and photoresist hinge drivers. Top: Asymmetric mirror assembly, fully rotated. Bottom: Detail of mechanical limiters.

$45^{\circ}$ mirrors, during and after rotation. Here, the design is almost symmetric, and both parts measure $1 \mathrm{~mm}$ parallel to the axis of rotation and $500 \mu \mathrm{m}$ orthogonal to it. However, exact symmetry is unnecessary, and asymmetric structures may also be assembled with precision as shown in Fig. 6. Here, the main mirror has dimensions $720 \mu \mathrm{m}$ orthogonal to the axis, and the stabilizing flap is reduced to $280 \mu \mathrm{m}$. These results suggest that gravitational torque and flap inertia are not significant in the assembly dynamics, which must instead be dominated by the viscosity of the meltable pads. Symmetry in the layout of the limiter does not appear necessary either; Fig. 7 shows an assembly with a base width of $250 \mu \mathrm{m}$, which has assembled correctly with a single limiter.

The viscosity of Hoechst AZ4562 is a strong function of temperature. Assembly took many hours at $130{ }^{\circ} \mathrm{C}$ and $5 \mathrm{~min}$ at $150{ }^{\circ} \mathrm{C}$. At intermediate temperatures, the melted hinge driver pads retained a semicylindrical shape, but above $150^{\circ} \mathrm{C}$, or if the samples were melted for too long, beads were formed. Fig. 8 shows the variation of rotation angle with heating time at $130{ }^{\circ} \mathrm{C}$ for a symmetric mirror assembly, measured using an optical microscope equipped with a Mitutuyo height gauge. This shows a gradual decrease in the rotation rate with time, from an initial value of $\approx 1 \% \mathrm{~min}$ and an accurate match between the rotation rates of the two flaps.

Because of the long lever arm, the accuracy of the mechanism is potentially high. However, even if the limiter mechanism operates correctly, errors in establishing the lengths $L$ and $L \sqrt{2}$ in Fig. 4 translate into angular errors in the final structure. Definition of the length $L$ depends on the variation in slot width caused by etching, while definition of the length
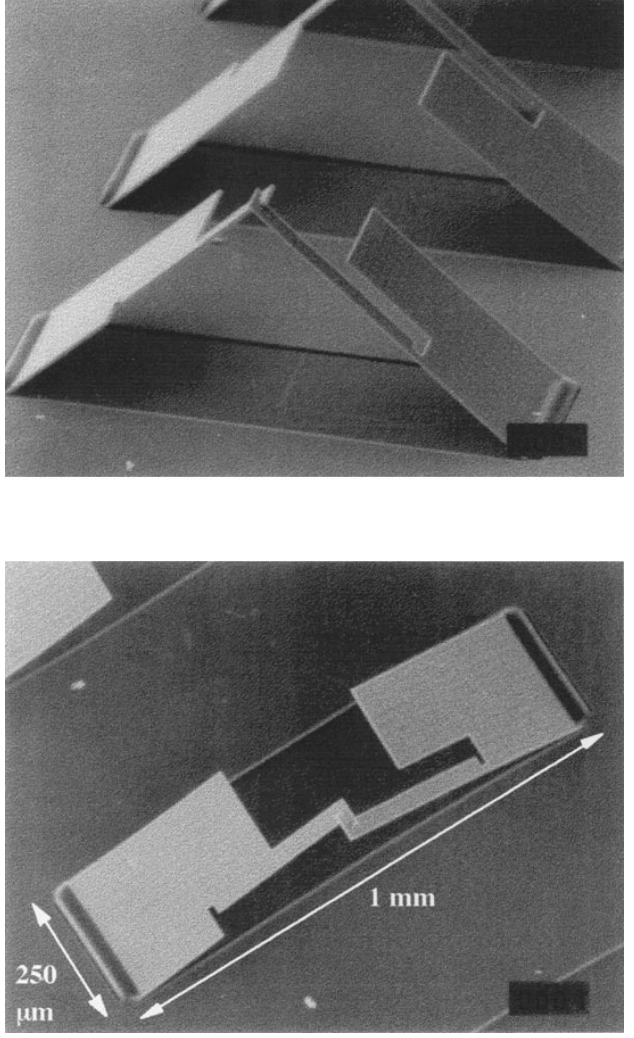

Fig. 7. Self-assembled structures based on BSOI parts and photoresist hinge drivers. Top: Oblique view of single-limiter mirror assembly, fully rotated. Bottom: Top view.

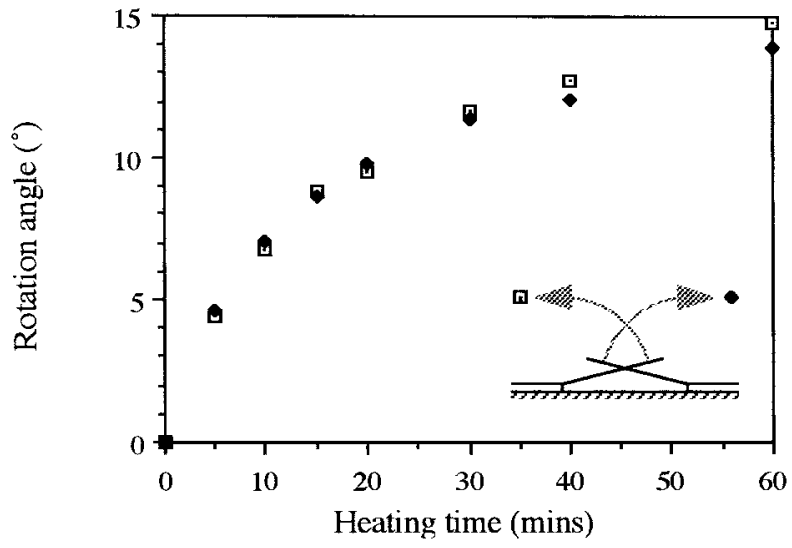

Fig. 8. Variation of rotation angle with heating time at $130{ }^{\circ} \mathrm{C}$ for $500-\mu \mathrm{m}$-wide symmetric mirror assemblies rotated by Hoechst AZ4562 photoresist.

$L \sqrt{2}$ depends on the degree to which the gap between the fixed and moving parts is closed during rotation. For example, in our process, slots widen by $\delta_{1} \approx 1 \mu \mathrm{m}$ on either side (due mainly to wet etching of the Cr mask). Similarly, SEM photographs show final separations $\delta_{2}$ between fixed and moving parts of up to $2 \mu \mathrm{m}$.

The angular assembly error may be estimated as $\Delta \theta=$ $45^{\circ}-\cos ^{-1}\left\{\left(L+\delta_{1}\right) /\left(L \sqrt{2}+\delta_{2}\right)\right\}$. Clearly, the twodimensional errors cause assembly errors of opposite sign. For example, for design dimensions of $L=500 \mu \mathrm{m}$ and errors of $\delta_{1}=1 \mu \mathrm{m}$ and $\delta_{2}=0$, we obtain $\Delta \theta=+0.12^{\circ}$, 
while for $\delta_{1}=0$ and $\delta_{2}=2 \mu \mathrm{m}, \Delta \theta=-0.16^{\circ}$. Both errors are small.

To verify these predictions, assembly accuracy was measured by back-reflection, using a HeNe laser and a rotation stage equipped with a Vernier gauge calibrated in $1^{\prime}$ steps. Mirror inclinations were generally slightly below their nominal value of $45^{\circ}$, so that $\Delta \theta$ is negative. The argument above suggests that overetching of the mechanical parts or incomplete rotation must be responsible for this error. For the symmetric mirror shown in Fig. 5, the rotational angles were $44^{\circ} 20^{\prime}$ (front face) and $44^{\circ} 10^{\prime}$ (back face). For the asymmetric assembly of Fig. 6, the corresponding results were $44^{\circ} 40^{\prime}$ (front face) and $44^{\circ} 52^{\prime}$ (back face). These results suggest that errors of $|\Delta \theta|<1^{\circ}$ may be routinely achieved. Improved accuracy would be obtained by eliminating the wet etch step used in defining the $\mathrm{Cr}$ hard mask. No significant creep in the structures has been observed over a period of some four months.

The process yield is currently modest $(\approx 20 \%$ of parts rotate; $5 \%$ rotate through $45^{\circ} \pm 1^{\circ}$ ). A major difficulty is the local lack of a stop-on-oxide dry etch. The $\mathrm{CHF}_{3} / \mathrm{O}_{2} / \mathrm{Ar}$ etch used erodes both silicon and silica, the latter at a rate $\approx 2.5$ times that of the former. Small variations in etch rate over the wafer are thus transformed into large variations in oxide etch depth, so that in some regions the oxide is not reached at all, while in others it is etched right through. Both errors cause catastrophic failures (the first, because parts are not separated, and the second because residual resist causes stick-down). Only if the oxide is just penetrated does the rest of the process operate correctly. More modern etches (such as the STS ASE process [38]) offer a simple solution. A further cause of low yield is detaching of parts during sacrificial layer release and freeze drying. We are currently studying methods of increasing resist adhesion.

For structures that assembled correctly, small differences between the rotation rates of the flaps were ascribed to two effects. Errors in mask overlay resulted in the lever arm of one hinge driver pad in a pair being lengthened at the expense of the other, as shown in Fig. 9(a). Alternatively, differences in structure-for example, the use of larger perforations in one flap-caused variations in resist height and an increase in the volume of one pad compared with the other, as in Fig. 9(b). In fact, Fig. 10 shows measured surface profiles of earlier part-fabricated catches made using $10-\mu \mathrm{m}$-thick BSOI. The perforations in Catch 2 (44 $\mu \mathrm{m}$ square) are much larger than those in Catch 1 (4 $\mu \mathrm{m}$ square) and are responsible for a reduction in pad height from 8 to $6 \mu \mathrm{m}$. For both types of error, the larger pad usually had an enhanced rotation rate, as shown in Fig. 9(c).

The total length of the hinge driver pads was not found to be particularly significant, provided they were of reasonable size compared with the mechanical parts. This result is consistent with earlier predictions of the dominance of surface tension force in the microstructure size domain [28]. Its significance lies in the implication that functional elements may be designed without fear that their layout will affect the assembly operation.

For example, Fig. 11 shows a $45^{\circ}$ resonant mirror scanner. The geometry is similar to Fig. 6, but the large mirror has been

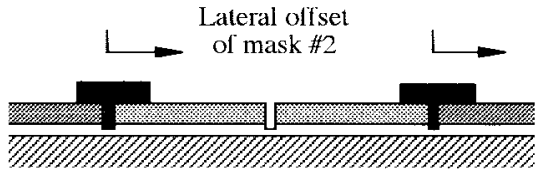

(a)

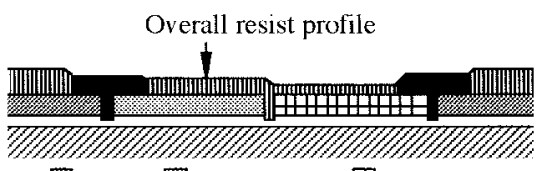

羿 Solid Small holes $\boxplus$ Large holes

(b)

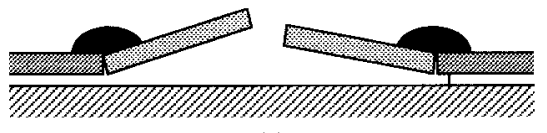

(c)

Fig. 9. (a) Overlay error and (b) resist thickness variation leading to (c) rotation asymmetry.

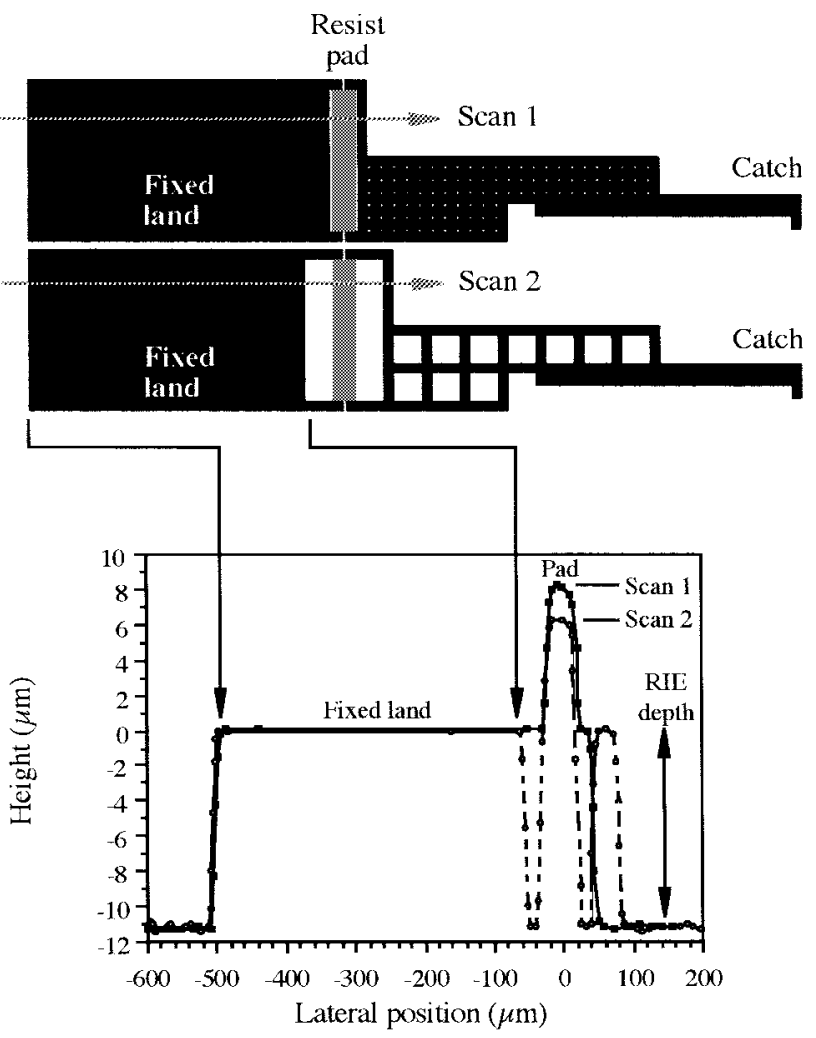

Fig. 10. Surface profiles obtained from part-completed structures in $10-\mu$ m-thick BSOI with small and large perforations, showing difference in resist hinge driver pad height.

cut away to form a frame carrying a smaller mirror on a torsion bar. The important feature here is that self-assembly has still occurred, despite the fact that one flap has been rotated by four hinge drivers, and the other by only two, confirming the lack of influence of gravitational forces. Torsional oscillation of the mirror is excited by a skewed set of interdigitated electrodes, with the fixed half of the comb located on the substrate and the moving half on the scanning mirror. Excellent alignment is maintained between the two halves of the drive, suggesting that precise assembly operations will be possible. 

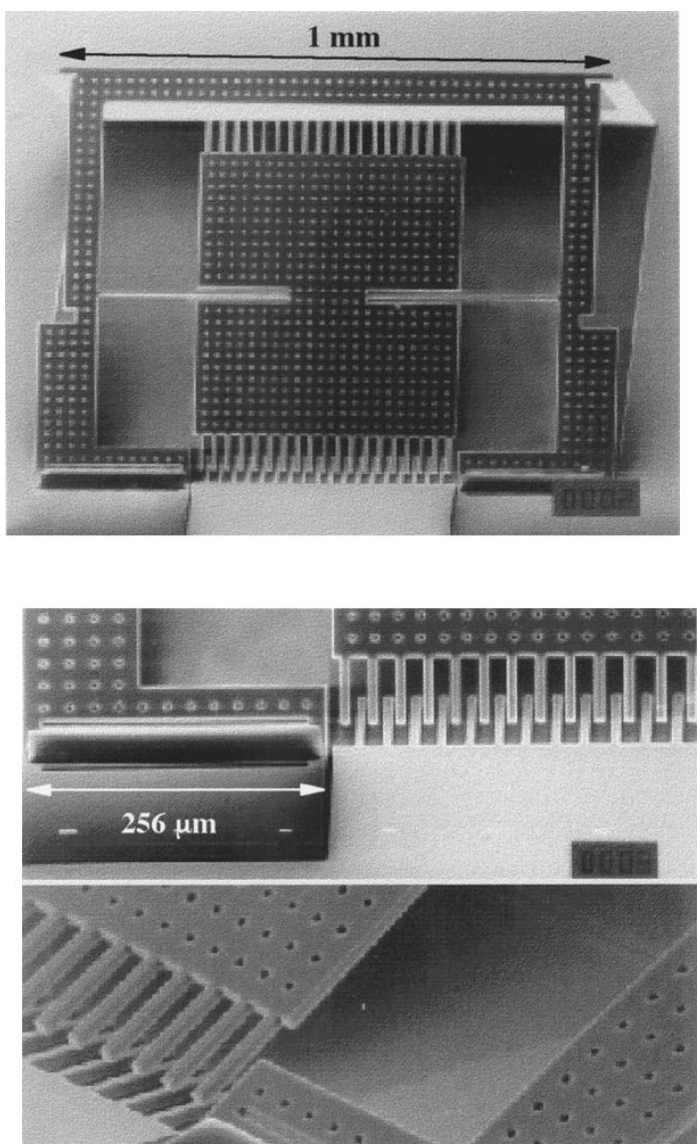

Fig. 11. Self-assembled 3-D electrostatic torsion mirror scanner based on BSOI parts and photoresist hinge drivers. Top: Complete structure. Bottom: Electrode details.

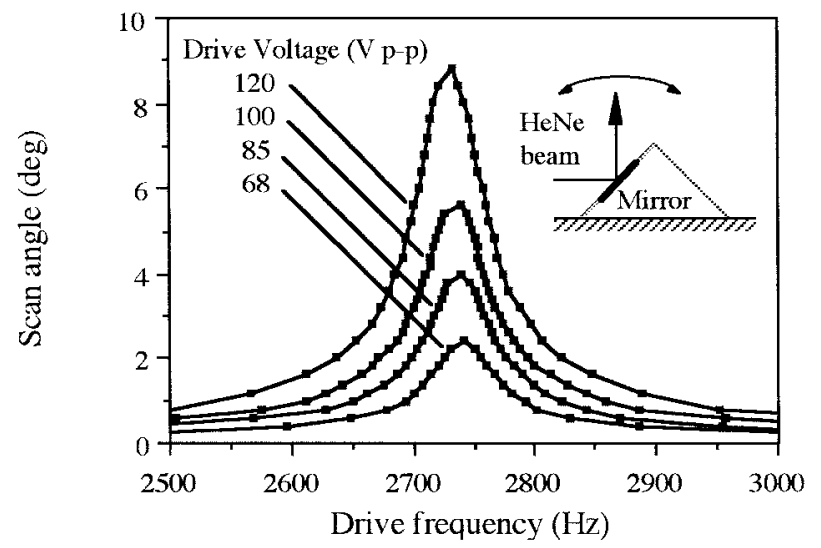

Fig. 12. Variation of optical scan angle with drive frequency for self-assembled 3-D torsion mirror assembly at various drive voltages.

The scanner was driven by a sinusoidal drive voltage and used to deflect a HeNe laser beam. Reasonable angular deflections were obtained for operation at normal atmospheric pressure, and mirror oscillation was observed without apparent motion of the framing structure. The drive voltage was large compared with previous designs [23] because of the relatively large electrode separation, and there is clearly scope for design optimization. Fig. 12 shows the variation of the optical scan angle (equal to twice the peak-to-peak mechanical deflection angle) with drive frequency at different drive voltages. There is a sharp resonance at $2725 \mathrm{~Hz}$, corresponding to a mechanical frequency of $5450 \mathrm{~Hz}$, and at this point a useful angular deflection close to $10^{\circ}$ was obtained at the largest drive voltage (120 V peak-to-peak).

\section{CONCLUSIONS}

We have developed a new low-temperature surface micromachining process for self-assembly of 3-D microstructures, based on mechanical parts formed from bonded silicon-oninsulator material that are rotated out-of-plane by the surface tension obtained by melting thick pads of photoresist. The fabrication sequence is extremely simple compared with most multilayer MEMS processes, involving just two lithography steps, one anisotropic dry etch step, one isotropic wet etch step, and a final freeze-dry release. Although they are not adjustable or reusable, the hinge drivers are extremely compact compared with other sources used to power out-of-plane rotation, such as linear vibromotors [11] or microengines [13], and are capable of assembling surprisingly large structures, in the millimetersize range.

A simple mechanism has been developed for the accurate limitation of $45^{\circ}$ rotations, with no increase in process complexity, based on the simultaneous rotation of a pair of flaps. Using this, a variety of 3-D micro-optomechanical structures have been demonstrated, and the main sources of inaccuracy in assembly have been identified. Accurate second-layer mask overlay and good planarization of the resist surface are the key requirements. The lack of a strong dependence on exact symmetry between the mechanical parts carried by the two flaps suggests that the technique may be useful for assembling many other different 3-D microstructures. Attention must now be given to the long-term stability and integrity of the melted resist pads.

\section{ACKNOWLEDGMENT}

The author is extremely grateful to BCO Technology (N. I.) Ltd. for preparing the BSOI material used in this study and to EPSRC for financial support.

\section{REFERENCES}

[1] K. Suzuki, I. Shimoyama, H. Miura, and Y. Ezura, "Creation of an insect-based microrobot with an external skeleton and elastic joints," in Proc. Micro Electro Mechanical Systems '92, Travemunde, Germany, Feb. 4-7, 1992, pp. 190-195.

[2] K. Suzuki, I. Shimoyama, and H. Miura, "Insect model based microrobot with elastic hinges" J. Microelectromech. Syst., vol. 3, pp. 4-9, 1994.

[3] M. G. Allen, M. Scheidl, and R. L. Smith, "Design and fabrication of movable silicon plates suspended by flexible supports," in Proc. IEEE Microelectromechanical Syst. Workshop, Feb. 1989, pp. 76-81.

[4] "Movable micromachined silicon plates with integrated position sensing" Sens. Actuators, vols. A21-23, pp. 211-214, 1990.

[5] L. S. Fan, Y.-C. Tai, R. S. Muller, "Integrated movable micromechanical structures for sensors and actuators," IEEE Trans. Electron Devices, vol. 35, pp. 724-730, 1988.

[6] K. S. Pister, M. W. Judy, S. R. Burgett, and R. S. Fearing, "Microfabricated hinges," Sens. Actuators, vol. A33, pp. 249-256, 1992.

[7] I. Shimoyama, O. Kano, and H. Miura, "3D microstructures folded by Lorentz force," in Proc. 11th Int. Workshop MicroElectroMechanical Systems, Heidelberg, Germany, Jan 25-29, 1998, pp. 24-28.

[8] T. Ebefors, E. Kälvesten, and G. Stemme, "New small radius joints based on thermal shrinkage of polyimide in V-grooves for robust selfassembly 3D microstructures," J. Micromech. Microeng., vol. 8, pp. 188-194, 1988 
[9] E. Smela, O. Inganä, and I. Lundströ, "Self-opening and closing boxes and other micromachined folding structures," in Proc. 8th Int. Conf. on Solid-State Sensors and Actuators, Stockholm, Sweden, pp. 350-351, June 25-29, 1995.

[10] _ "Controlled folding of micrometer-size structures" Science, vol. 268, pp. 1735-1738, 1995.

[11] N. J. Daneman, N. C. Tien, O. Solgaard, A. P. Pisano, K. Y. Lau, and R. S. Muller, "Linear vibromotor for positioning optical components," J. Microelectromech Syst., vol. 5, pp. 159-165, 1996.

[12] N. C. Tien, O. Solgaard, M. H. Kiang, M. Daneman, K. Y. Lau, and R. S. Muller, "Surface micromachined mirrors for laser-beam positioning" Sens. Actuators, vol. A52, pp. 76-80, 1996.

[13] E. J. Garcia and J. J. Sniegowski, "Surface micromachined microengine," Sens. Actuators, vol. A48, pp. 203-214, 1995.

[14] E. J. Garcia, "Micro-flex mirror and instability actuation technique," in Proc. 11th Int. Workshop MicroElectro Mechanical Systems, Heidelberg, Germany, Jan 25-29, 1998, pp. 470-475.

[15] L. Y. Lin, S. S. Lee, K. S. J. Pister, and M. C. Wu, "Micromachined micro-optical bench for optoelectronic packaging," in Proc. 7th Annual Meet. IEEE Lasers and Electro-Optics Soc., Boston, MA, Oct. 31-Nov. 3, 1994, pp. 219-220.

[16] , "Three-dimensional micro-Fresnel optical elements fabricated by micromachining techniques," Electron. Lett., vol. 30, pp. 448-449, 1994.

[17] S. S. Lee, L. Y. Lin, K. S. J. Pister, M. C. Wu, H. C. Lee, and P. Grodzinski, "Passively aligned hybrid integration of $8 \times 1$ micromachined micro-Fresnel lens arrays and $8 \times 1$ vertical-cavity surface-emitting laser arrays for free-space optical interconnect," IEEE Photon. Technol. Lett., vol. 7, pp. 1031-1033, 1995.

[18] M. C. Wu, L.-Y. Lin, S.-S. Lee, and K. S. J. Pister, "Micromachined free-space integrated micro-optics," Sens. Actuators, vol. A50, pp. 127-134, 1995.

[19] C. R. King C.R., L. Y. Lin, M. C. Wu, "Out-of-plane refractive microlens fabricated by surface micromachining," IEEE Photon. Technol. Lett., vol. 8, pp. 1349-1351, 1996.

[20] J. L. Shen, L. Y. Lin, S. S. Lee, M. C. Wu, and M. Sergent, "Surfacemicromachined tunable three-dimensional solid Fabry-Perot etalons with dielectric coatings," Electron. Lett., vol. 31, pp. 2172-2173, 1995.

[21] L. Y. Lin, J. L. Shen, M. C. Wu, and A. M. Segent, "Tunable three-dimensional solid Fabry-Perot etalons fabricated by surface micromachining," IEEE Photon. Technol. Lett., vol. 8, pp. 101-103, 1996.

[22] M.-H. Kiang, O. Solgaard, R. S. Muller, and K. Y. Lau, "Micromachined polysilicon microscanners for barcode readers," IEEE Photon. Technol. Lett., vol. 8, pp. 95-97, 1996.

[23] M.-H. Kiang, O. Solgaard, and K. Y. Lau, "Electrostatic combdriveactuated micromirrors for laser-beam scanning and positioning," $J$. Micromech. Syst., vol. 7, pp. 27-37, 1998.

[24] J. R. Reid, V. M. Bright, and J. H. Comtois, "Arrays of thermal microactuators coupled to micro-optical components," in Proc. SPIE, 1996, vol. 2865, pp. 74-82.

[25] J. T. Butler, V. M. Bright, and J. R. Reid, "Scanning and rotating micromirrors using thermal actuators" in Proc. SPIE, 1997, vol. 3131, pp. 134-144.
[26] T. Ebefors, E. Kälvesten, and G. Stemme, "Dynamic actuation of polyimide V-groove joints by electrical heating," Sens. Actuators, vol. A67, pp. 199-204, 1998.

[27] M. C. Wu, "Micromachining for optical and optoelectronic systems,', Proc. IEEE, vol. 85, pp. 1833-1856, 1997.

[28] R. R. A. Syms and E. M. Yeatman, "Self-assembly of fully threedimensional microstructures using rotation by surface tension forces," Electron. Lett., vol. 29, pp. 662-664, 1993.

[29] R. R. A. Syms, "Equilibrium of hinged and hingeless structures rotated using surface tension forces," J. Microelectromech. Syst., vol. 4, pp. 177-184, 1995.

[30] K. F. Harsh, R. S. Irwin, and Y. C. Lee, "Solder self-assembly for MEMS," in Proc. 4th Int. Instrumentation Symp., Reno, NV, May 3-7, 1998, pp. 256-261.

[31] K. Harsh and Y. C. Lee, "Modeling for solder self-assembled MEMS," in Proc. SPIE, vol. 3289, San Jose, CA, Jan. 24-30, 1998, pp. 177-184.

[32] P. W. Green, R. R. A. Syms, and E. M. Yeatman, "Demonstration of three-dimensional microstructure self-assembly," J. Microelectromech. Syst., vol. 4, pp. 170-176, 1995.

[33] R. R. A. Syms, "Rotational self-assembly of complex microstructures by the surface tension of glass," Sens. Actuators, vol. A65, pp. 238-243, 1998.

[34] A. Benitez, J. Esteve, and J. Bausells, "Bulk silicon microelectromechanical devices fabricated from commercial bonded and etched-back silicon-on-insulator substrates," Sens. Actuators, vol. A50, pp. 99-103, 1995.

[35] E. H. Klaassen, K. Petersen, J. M. Noworolski, J. Logan, N. I. Maluf, J. Brown, C. Storment, W. McCulley, and T. A. Kovacs, "Silicon fusion bonding and deep reactive ion etching: A new technology for microstructures," Sens. Actuators, vol. A52, pp. 132-139, 1996.

[36] R. Yeh, E. J. J. Kruglick, and K. S. J. Pister, "Surface-micromachined components for articulated microrobots," J. Microelectromech. Syst., vol. 5, pp. 10-17, 1996.

[37] A. Friedberger and R. S. Müller, "Improved surface-micromachined hinges for fold-out structures," J. Microelectromech. Syst., vol. 7, pp. 315-319, 1998

[38] J. K. Bhardwaj and H. Ashraf, "Advanced silicon etching using high density plasmas," in Proc. SPIE, vol. 2639, pp. 224-233, 1995.

Richard R. A. Syms (M'98) was born in Norfolk, VA, in 1958. He received the B.A. degree in engineering science and the D.Phil. degree from Worcester College, Oxford, U.K., in 1979 and 1982, respectively.

His doctoral work was on volume holographic optical elements. He has been Head of the Optical and Semiconductor Devices Section in the Department of Electrical and Electronic Engineering, Imperial College London, U.K., since 1992 and Professor of microsystems technology since 1996. He has published approximately 80 papers and two books on holography, integrated optics, and microengineering. His current interests are silica-on-silicon integrated optics and silicon-based MEMS. Most recently, he has been developing microengineered mass spectrometers, frequency-tunable microactuators, and three-dimensional self-assembling microstructures. 